\title{
POSTER: Basebads: Automated Security Analysis of Baseband Firmware
}

\author{
Grant Hernandez \\ University of Florida \\ Gainesville, FL, USA \\ grant.hernandez@ufl.edu
}

\author{
Kevin R. B. Butler \\ University of Florida \\ Gainesville, FL, USA \\ butler@ufl.edu
}

\begin{abstract}
Mobile devices are more connected than ever before through the use of multiple wireless protocols, including the $2 \mathrm{G}, 3 \mathrm{G}$, and $4 \mathrm{G}$ cellular standards. To manage and interact with cellular networks, phones use dedicated and highly proprietary baseband processors running custom, closed-source firmware. Despite the increasing complexity of modern cellular standards, there is no reference implementation, leading individual baseband manufacturers to create their own in-house versions. The proprietary nature of baseband firmware combined with the complexity of standards has created a barrier for researchers to comprehensively audit the security of these implementations. To address this, we present SPIKERXG, an extensible, baseband testing platform that employs firmware instrumentation to intelligently target protocol messages.
\end{abstract}

\section{CCS CONCEPTS}

- Security and privacy $\rightarrow$ Mobile platform security; • Computer systems organization $\rightarrow$ Firmware; $\bullet$ Networks $\rightarrow$ Protocol testing and verification;

\section{KEYWORDS}

Basebands; Mobile; Fuzzing

\section{INTRODUCTION}

As mobile devices became ubiquitous in modern society, so did the demand for improved mobility. Since the transition from first-generation (1G) pure analog signaling to digital (2G) systems, cellular standards have successively improved data rates, operational range, and overall reliability. To provide this level of service, network operators have adopted new infrastructure and purchased additional resources. On the mobile device side, each generational change has required a corresponding leap forward in processing power and signal processing. Today, cellular functions on smartphones are realized through dedicated cellular processors, also known as basebands. These basebands are specialized, stand-alone, chips that implement the $2 \mathrm{G}, 3 \mathrm{G}, 4 \mathrm{G}$, and upcoming $5 \mathrm{G}$ standards. As such, they run complex embedded software, known as firmware. This firmware is critical to mobile device functionality as it is responsible for decoding and encoding all over-the-air messages, managing calls, exchanging text messages,

Permission to make digital or hard copies of part or all of this work for personal or classroom use is granted without fee provided that copies are not made or distributed for profit or commercial advantage and that copies bear this notice and the full citation on the first page. Copyrights for third-party components of this work must be honored. For all other uses, contact the owner/author(s).

WiSec '19, May 15-17, 2019, Miami, FL, USA

(C) 2019 Copyright held by the owner/author(s)

ACM ISBN 978-1-4503-6726-4/19/05.

https://doi.org/10.1145/3317549.3326310 and establishing data connections. Given the responsibilities of baseband firmware, its integrity is also critical protecting sensitive data residing on the device and preventing against wide-scale attacks [10]. Unfortunately, most if not all commercial baseband firmware is proprietary and closed source, slowing down external security assessments. Worse, what little previous research that exists has taken an ad-hoc approach to baseband security, leaving many questions unanswered. These barriers and limited approaches have stymied research into the overall trustworthiness of baseband processors and their firmware.

To fill this shortcoming, we developed a baseband testing framework, SPIKERXG. Our framework aims to test many baseband implementations through over-the-air protocol manipulation and firmware introspection. By understanding how basebands fail across vendors and cellular protocols, we can understand the overall security posture of baseband firmware.

\section{BACKGROUND \& RELATED WORK}

Every modern cell phone has a chip referred to as a baseband, modem, or cellular processor. This is responsible for handling low-level, overthe-air messages of the underlying cellular protocol. Basebands are responsible for functions like attaching and detaching to a base station and sending/receiving calls, SMS messages, and data. Application processors have been hardened significantly in recent years against exploits. Countermeasures such as address space layout randomization and non-executable memory have been employed to defend against various types of exploitation. Cellular processors, however, may not have the same mitigations, making them vulnerable to remote attack. Each manufacturer, such as Qualcomm, Samsung, and Intel have their own firmware, which is proprietary. These various interpretations of the cellular protocol are likely littered with undiscovered bugs due to the barrier to entry for baseband research.

One of the earliest works by Mulliner et al. [7] demonstrated a method to generate invalid SMS messages on a local device to test its robustness. This work was extended to illustrate that remote SMS attacks against mobile devices are possible [6]. The following work demonstrated that even more damaging remote attacks are possible over-the-air due to baseband memory corruption $[8,12]$. This paper highlighted the relative insecurity of baseband processors when compared to application processors, due to their lack of hardening against memory corruption. Unfortunately, this work was limited to two phones and does not capture the whole baseband space. Work similar to SPIKERXG [11] tests device GSM implementations, but only focuses on SMS and cell broadcast traffic. No systematic approach is taken. T-Fuzz [3] takes a model-based approach to telecommunication protocol testing but fails to categorize the found unwanted behavior, leading to a lack of understanding of real impact. Recent papers on 


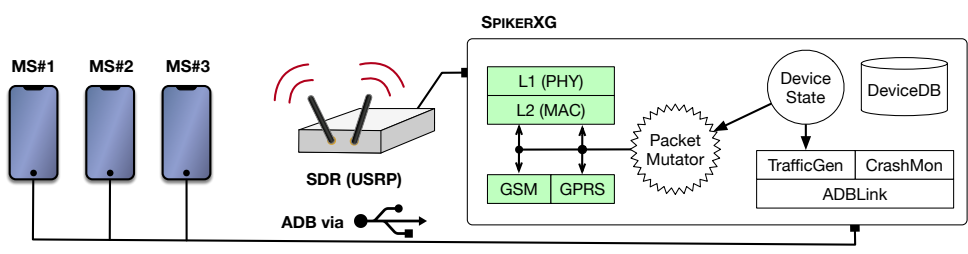

Figure 1: A high-level diagram of SPIKERXG and its components.

LTE implementations $[2,4,9]$ typically focus on the protocol state machine level and may not capture all classes of implementation flaws or speak to exploitability. The most similar approach to SPIKERXG [1] creates an emulated environment for device testing but does not discuss the root causes of the issues found.

\section{METHODOLOGY \& PROPOSED WORK}

In this section, we begin with an overview of SPIKERXG and then describe the design and implementation. As shown in Figure 1 SPIKERXG creates a feedback loop with a cellular base station and target devices to automatically test baseband processors for implementation flaws. The methodology of SPIKERXG is independent of any individual implementation of a cellular protocol, but as a proof of concept, we apply our approach to the $2 \mathrm{G}$ protocol. For our cellular base station backend, we chose YateBTS. The core of Yate is written in C++ and SPIKERXG in Python 3.7 for portability and ease of development. SPIKERXG connects to YateBTS through an instrumented control and data path, which allows for the base station to be remotely administered and for over-the-air packets to be inspected or modified by SPIKERXG logic. These control and data paths are created via persistent socket connections for speed.

At its core, SPIKERXG mutates packets leaving the base station to travel through the air. Our system completely mediates all packets leaving the base station (downlink), with the exception of any multicast control channels, such as the Broadcast Control Channel $(\mathrm{BCCH})$. We avoided mutating packets on broadcast channels due to difficulty in suppressing them from innocent devices. Instead, we targeted PDUs directed to a single device (unicast). As such, we focus on fuzzing GSM and GPRS Layer 3 (L3) message frames, which require a Layer 2 (L2) connection to be established to a single device. To detect and manage devices without user interaction our system incorporates device feedback in the form of logs and packets. To meet the goal of automatically fuzzing devices over-the-air, the fuzzing portion of SPIKERXG must operate with knowledge of the device state. This is crucial for detecting device anomalies such as modem crashes, reboots, hangs, and other runtime errors.

Using SPIKERXG we were able to simultaneously fuzz multiple Android devices over-the-air, leading to the discovery of two remote crashes in the $2 \mathrm{G}$ protocol implementations for the Intel and Exynos baseband processors. The affected phones were an ASUS ZenPhone 2 and Samsung S7 EDGE. These crashes caused a complete device reboot and were found in less than six hours of fuzzing.

Our initial proof of concept with SPIKERXG was solely focused on dynamic testing, but this approach has limitations in the types of flaws that can be automatically found. As such, to extend this work we will focus on scaling up the testing process by focusing on the baseband firmware itself. To target the firmware, we will use AVATAR $^{2}$ [5], which interoperates well with QEMU and popular symbolic execution and binary analysis frameworks. SPIKERXG will create basebandspecific machine and interface definitions in order to inject over-theair packets directly into the firmware. This will be challenging and require the use of reverse engineering tools to determine the firmware format and I/O. The benefit of this approach is that, unlike purely dynamic testing, symbolic execution and other powerful techniques can be employed. This will serve to get a more detailed view of program flaws in order to determine their exploitability and their reproducibility in a real setting.

\section{REFERENCES}

[1] Kaiming Fang and Guanhua Yan. 2018. Emulation-Instrumented Fuzz Testing of 4G/LTE Android Mobile Devices Guided by Reinforcement Learning. In Computer Security (European Symposium on Research in Computer Security), Javier Lopez, Jianying Zhou, and Miguel Soriano (Eds.). Springer International Publishing, 2040.

[2] Syed Rafiul Hussain, Omar Chowdhury, Shagufta Mehnaz, and Elisa Bertino. 2018. LTEInspector: A Systematic Approach for Adversarial Testing of 4G LTE. In Symposium on Network and Distributed Systems Security (NDSS). 18-21.

[3] W. Johansson, M. Svensson, U. E. Larson, M. Almgren, and V. Gulisano. 2014. T-Fuzz: Model-Based Fuzzing for Robustness Testing of Telecommunication Protocols. In Verification and Validation 2014 IEEE Seventh International Conference on Software Testing. 323-332. https://doi.org/10.1109/ICST.2014.45

[4] Hongil Kim, Jiho Lee, Lee Eunkyu, and Yongdae Kim. 2019. Touching the Untouchables: Dynamic Security Analysis of the LTE Control Plane. In Proceedings of the IEEE Symposium on Security \& Privacy (SP). IEEE.

[5] Marius Muench, Jan Stijohann, Frank Kargl, Aurélien Francillon, and Davide Balzarotti. 2018. What you corrupt is not what you crash: Challenges in fuzzing embedded devices. In NDSS 2018, Network and Distributed Systems Security Symposium, 18-21 February 2018, San Diego, CA, USA. San Diego, UNITED STATES. http://www.eurecom.fr/publication/5417

[6] Collin Mulliner, Nico Golde, and Jean-Pierre Seifert. 2011. SMS of Death: From Analyzing to Attacking Mobile Phones on a Large Scale. In Proceedings of the 20th USENIX Conference on Security (SEC'11). USENIX Association, Berkeley, CA USA, 24-24. http://dl.acm.org/citation.cfm?id=2028067.2028091

[7] Collin Mulliner and Charlie Miller. 2009. Fuzzing the Phone in your Phone. In Black Hat USA, Vol. 25. 31.

[8] Nico Golde. 2018. There's Life in the Old Dog Yet: Tearing New Holes into Intel/iPhone Cellular Modems. (April 2018). https: //comsecuris.com/blog/posts/theres_life_in_the_old_dog_yet_tearing_new holes_into_inteliphone_cellular_modems/ Comsecuris Blog.

[9] David Rupprecht, Kai Jansen, and Christina Pöpper. 2016. Putting LTE Security Functions to the Test: A Framework to Evaluate Implementation Correctness. In Proceedings of the 10th USENIX Conference on Offensive Technologies (WOOT'16). USENIX Association, Berkeley, CA, USA, 40-51. http://dl.acm.org/citation.cfm? id=3027019.3027023

[10] Patrick Traynor, Michael Lin, Machigar Ongtang, Vikhyath Rao, Trent Jaeger, Patrick McDaniel, and Thomas La Porta. 2009. On Cellular Botnets: Measuring the Impact of Malicious Devices on a Cellular Network Core. In Proceedings of the 16th ACM Conference on Computer and Communications Security (CCS '09). ACM, New York, NY, USA, 223-234. https://doi.org/10.1145/1653662.1653690

[11] Fabian van den Broek, Brinio Hond, and Arturo Cedillo Torres. 2014. Security Testing of GSM Implementations. In Engineering Secure Software and Systems (Lecture Notes in Computer Science), Jan Jürjens, Frank Piessens, and Nataliia Bielova (Eds.). Springer International Publishing, 179-195.

[12] Ralf-Philipp Weinmann. 2012. Baseband Attacks: Remote Exploitation of Memory Corruptions in Cellular Protocol Stacks.. In WOOT. 12-21. 